\title{
Effect of ADRA2A gene polymorphisms on the anesthetic and analgesic effects of dexmedetomidine in Chinese Han women with cesarean section
}

\author{
ZHIMEI FU, BINGWEI HU, TINGTING MA, QIANDONG WANG and DONGDONG WANG \\ Department of Anesthesiology, Tongde Hospital of Zhejiang Province, Hangzhou, Zhejiang 310012, P.R. China
}

Received September 22, 2018; Accepted August 8, 2019

DOI: $10.3892 /$ etm.2020.8481

\begin{abstract}
It is well known that differences in drug reactions among individuals are widespread, and therefore the study of genetic polymorphisms of drug targets has become a research hotspot. Dexmedetomidine is clinically effective by acting on $\alpha 2$ adrenergic receptor and the impact of the adrenoceptor $\alpha 2 \mathrm{~A}$ gene (ADRA2A) polymorphisms on the anesthetic and analgesic effects of dexmedetomidine is related to the clinical application of dexmedetomidine. The present study aimed to analyze the effects of the rs1800035, rs201376588 and rs775887911 locus single-nucleotide polymorphisms of the ADRA2A on the anesthetic and analgesic effects of dexmedetomidine in Chinese Han women. A total of 434 Chinese women undergoing cesarean section were enrolled in this study. A 3-ml fasting venous blood sample was collected from all subjects for genomic DNA extraction and genotype detection. The pre-anesthetic and post-anesthetic pain threshold (PTh), pain tolerance threshold (PTTh), mean arterial pressure, heart rate, blood oxygen saturation, cortisol (Cor) content, blood glucose (Glu) content, opioid usage, patient-controlled analgesia pressing times, surgical satisfaction and postoperative adverse reactions were recorded. The visual analogue scale (VAS) and Ramsay sedation score were evaluated. PTh and PTTh in the wild-type women were higher than those in the women with mutations $(\mathrm{P}<0.05)$. The postoperative VAS scores of wild-type women were lower than those of mutants $(\mathrm{P}<0.05)$. The Ramsay sedation scores of wild-type patients at $12 \mathrm{~h}$ after the operation were significantly higher than in those with mutations $(\mathrm{P}<0.05)$. The levels of Cor and Glu in women with mutations were significantly higher than those of wild-type women at 12, 24 and $48 \mathrm{~h}$ after surgery $(\mathrm{P}<0.05)$. The satisfaction with surgery of wild-type patients
\end{abstract}

Correspondence to: Dr Dongdong Wang, Department of Anesthesiology, Tongde Hospital of Zhejiang Province, 234 Gucui Road, Hangzhou, Zhejiang 310012, P.R. China E-mail: gregwangd@163.com

Key words: G protein-coupled receptors, cesarean section, dexmedetomidine, postoperative analgesia, single-nucleotide polymorphisms was higher than that of patients with mutations $(\mathrm{P}<0.05)$. Gene mutations of rs1800035, rs201376588 and rs775887911 loci in the ADRA2A gene reduced the anesthetic and analgesic effect during and after cesarean section in Chinese Han women. Postoperative analgesia of mothers with mutations may require higher doses of analgesics.

\section{Introduction}

The high rate of caesarean section has been a medical and social problem in China for many years. Since 1985, the international medical community has always considered the 'ideal' rate of cesarean section to be between 10 and $15 \%$ (1); the rate of caesarean section in China is far higher than this level, and in some areas, it is up to $90 \%$ (2). After cesarean section, pain stimulation can cause a series of pathophysiological changes, which not only brings great pain to the puerperae but also affects the functions of the respiratory, digestive, circulatory, endocrine and immune systems of the puerperae, and produces postoperative complications (3).

Dexmedetomidine is a highly selective $\alpha 2$ adrenergic receptor ( $\alpha 2-\mathrm{AR})$ agonist that is clinically used in sedation (4). A previous study also found that postoperative intravenous infusion of dexmedetomidine hydrochloride can improve the relief of postoperative acute pain and reduce opioid dosage (5). Recent studies have shown that dexmedetomidine use, combined with opioid use, in analgesia after cesarean section can not only reduce the consumption of analgesic drugs but can also reduce the pressing times for patient-controlled analgesia, reduce the incidence of postoperative complications and improve patient satisfaction with postoperative analgesia (6). However, previous research has found that the opioid analgesic effect is influenced by genetic factors (7), and that there is substantial variation in the effect of drug application between individuals. Therefore, the study of genetic factors has important clinical significance for the effect of dexmedetomidine on anesthesia and analgesia in cesarean section. The $\alpha 2-\mathrm{AR}$ is a receptor for dexmedetomidine, and whether its genetic polymorphisms affect the efficacy of dexmedetomidine is still unclear.

The adrenoceptor alpha 2A gene (ADRA2A)-coded $\alpha 2-A R$ protein is a member of the $G$ protein coupling receptor subfamily and consists of three highly homologous subtypes, $\alpha \mathrm{A}, \alpha \mathrm{B}$ and $\alpha 2 \mathrm{C}$, which regulate neurotransmitters released 
by the sympathetic and adrenergic neurons. These subtypes play an important role in the regulation of the central nervous system (8). The SNPs were analyzed to determine their influence on the anesthestic and analgesic effects of dexmedetomidine in Chinese Han women. From National Center for Biotechnology Information (NCBI; https://www. ncbi.nlm.nih.gov/snp/) single nucleotide polymorphism database, it was found that the $\mathrm{C} / \mathrm{G}$ mutation at rs1800035 leads to the mutation of Asn amino acid into Lys amino acid (9), the C/T mutation at rs201376588 (https://www.ncbi.nlm.nih. gov/snp/rs201376588\#variant_details) leads to the mutation of Arg amino acid into Cys, and the $\mathrm{C} / \mathrm{T}$ mutation at rs775887911 (https://www.ncbi.nlm.nih.gov/snp/rs775887911) leads to the mutation of Pro amino acid into Leu. It is not clear whether these three types of mutations lead to the alteration of amino acid sequence and the structural and functional changes of $\alpha 2$-ARs.

The present study aimed to investigate the effect of ADRA2A rs1800035, rs201376588 and rs775887911 SNPs on dexmedetomidine, which provides a theoretical basis for improving the administration of dexmedetomidine.

\section{Materials and methods}

General information. From May 2013 to October 2017, 434 Chinese Han women who underwent caesarean section under combined spinal epidural anesthesia in the Tongde Hospital of Zhejiang Province (Zhejiang province) were selected (Fig. 1), who were in line with the American Society of Anesthesiologists grading I-II; mean age, 29.5 \pm 3.9 years, range, 20-36 years; body weight, $58-82 \mathrm{~kg}$; body mass index (BMI) $27.2 \pm 3.5 \mathrm{~kg} / \mathrm{m}^{2}$ (average BMI, $27.2+3.5 \mathrm{~kg} / \mathrm{m}^{2}$ ); and gestational age, 38-40 weeks. Exclusion criteria were as follows: Congenital heart and lung disease, spinal canal anesthesia contraindication, hypertension or gestational hypertension, hyperthyroidism or hypothyroidism, diabetes or gestational diabetes mellitus. The present study was approved by the medical ethics committee of the Tongde Hospital of Zhejiang Province, (Zhejiang, China) and all subjects provided informed consent.

Methods. The anesthesia used within the present study consisted of an intrathecal injection of $10 \mathrm{mg}$ bupivacaine and $4 \mathrm{mg}$ dexmedetomidine. The epidural analgesia was performed at the L2-3 interspace by an 18-gauge Tuohy needle using the method of loss of resistance to air in the left lateral position. After the epidural puncture was successful, the epidural puncture needle was used as a guide. A 25G lumbar puncture needle was inserted, and the arachnoid was pierced through the epidural puncture needle. The needle core was withdrawn and the cerebrospinal fluid was discharged from the needle. A volume of $2-3 \mathrm{ml}$ of $0.5 \%$ ropivacaine and $0.5 \mu \mathrm{g} / \mathrm{ml}$ dexmedetomidine was injected via the spine, and a lumbar puncture needle was inserted into the epidural catheter via an epidural needle. The epidural needle was withdrawn, the puncture point was covered with a sterile gauze, and the catheter was fixed.

Methods of analgesia. Ropivacaine hydrochloride $(0.1 \%)$ and $0.5 \mu \mathrm{g} / \mathrm{kg}$ dexmedetomidine hydrochloride were diluted in $0.9 \%$ normal saline to $100 \mathrm{ml}$, and epidural continuous auto-control analgesia was provided. When the mean arterial pressure of the puerpera was $<60 \mathrm{mmHg}, 10 \mathrm{mg}$ ephedrine was injected intravenously, and $0.2 \mathrm{mg}$ atropine sulfate was injected intravenously at a heart rate $(\mathrm{HR})<50 \mathrm{bpm}$. Epidural $10 \mathrm{ml}$ of $1 \%$ lidocaine was administrated to rescue if visual analogue score (VAS) was $>3$ after 30 min of epidural injection.

Indicators for observation and evaluation. Maternal basic information including maternal age, gestational age, height, weight and operation time, was assessed. When each patient entered the operating room, vital signs were continuously monitored by an Agilent V24C monitor (Agilent Technologies, Inc.) before anesthesia and 5, 10 and $30 \mathrm{~min}$ after anesthesia. Mean arterial pressure (MAP), HR and blood oxygen saturation $\left(\mathrm{SpO}_{2}\right)$ were monitored in the puerperae after surgery. A pain threshold (PTh) detector (EP601C PTh detector; Teaching Equipment Factory of Shanghai East China Normal University) was used to monitor the PTh and the pain tolerance threshold (PTTh) of the women before and after anesthesia. Measurements were taken before administration of the anesthetic and $1 \mathrm{~h}$ after administration. The PTh and PTTh were measured by a pain threshold detector using a microwave current stimulation method, and the two electrodes were fixed on the deltoid muscle of the left upper arm $1.5 \mathrm{~cm}$ apart. The detector produced a $50 \mathrm{~Hz}$ electrical stimulation with a pulse width of $0.5 \mathrm{msec}$. The current intensity gradually increased from 0 to $5 \mathrm{~mA}$. When the pain was first felt, the measured value was the PTh, and when the pain became unbearable, the measured value was the PTTh. The degree of pain caused by cesarean section at $6,12,24$ and $48 \mathrm{~h}$ after the operation was assessed by the VAS $(10,11)$. The VAS ranges between 0 and 10; 0 points indicates no pain; a score of 1-3 indicates mild pain; a score of 4-6 indicates moderate pain; and a score of 7-10 indicates severe pain. The sedation score at $6,12,24$ and $48 \mathrm{~h}$ after the operation was evaluated by the Ramsay sedation score system (12). Level 1 indicates anxiety or irritability; level 2 indicates that the patient is cooperative, quiet, and well directed; level 3 means drowsiness, but responds to instructions; level 4 indicates a quick response to tapping the brow or strong sound stimulation; level 5 indicates the patient is unresponsive to tapping the brow or strong sound stimulation; and level 6 indicates that the patient has no reaction to tapping the brow or strong sound stimulation. A sample of $4 \mathrm{ml}$ fasting venous blood was taken before the operation and at 6,12, 24 and $48 \mathrm{~h}$ after the operation. After standing for $20 \mathrm{~min}$, the serum was separated and stored at $-20^{\circ} \mathrm{C}$. The stress response of the patient before anesthesia and 6, 12, 24 and $48 \mathrm{~h}$ after surgery was detected by cortisol (Cor; cat. no. SKGE008B; R\&D Systems, Inc.) and blood glucose (Glu) detection kits (cat. no. ml076792; Shanghai Enzyme-linked Biotechnology Co., Ltd.). A satisfaction questionnaire of the analgesic effect was used to investigate the satisfaction of the puerperae with the analgesic effect, including 'very satisfied' (comfort), 'satisfaction' (no obvious discomfort), 'general' (mild discomfort, but not obvious) and 'not satisfied' (with obvious discomfort). The following formula was used: Satisfaction rate=(very satisfied + satisfaction)/total number of participants $\mathrm{x} 100 \%$. The side effects of using narcotic analgesics are well documented (13). The 
primary outcome indicators in this study were the PTh, PTTh, VAS and Ramsay scores. Secondary outcome indicators were Cor and Glu levels, and maternal satisfaction with cesarean delivery.

SNP locus selection. The drug target predicted by the Kyoto Encyclopedia of Genes and Genomes (KEGG) Pathway database showed that the target of dexmedetomidine was the ADRA2A. Based on the prediction of the influence of ADRA2A single-nucleotide polymorphism (SNP) site mutations on the protein structure and function in the Ensembl database (http://asia.ensembl.org/index.html), three loci were selected (rs1800035, rs20137658 and rs775887911) that had a great influence on protein structure and function.

Genotyping. Genomic DNA was extracted from $2 \mathrm{ml}$ of venous blood from each participant using a QIAamp DSP DNA Blood Mini kit (cat. no. 51104; Qiagen GmbH). The genotypes of the rs1800035, rs201376588 and rs775887911 loci were analyzed by PCR/Sanger sequencing. PCR products were purified after PCR amplification and then analyzed for each locus genotype by Sanger sequencing (Fig. 2). The primer sequences for PCR amplification at the rs1800035 site were as follows: Forward primer, CCTGGCTTAATTTTCCAGGCT and reverse primer, GAGTCCTGCCATCGCCTTTA (primer melting temperature $\left.(\mathrm{Tm}), 59^{\circ} \mathrm{C}\right)$. The primer sequences for PCR amplification at the rs201376588 site were as follows: Forward primer, CTGGCAGCAGGCCCAG and reverse primer, CATGGGCGCAAAGCTGC $\left(\mathrm{Tm}, 60^{\circ} \mathrm{C}\right)$. The primer sequences for PCR amplification at the rs775887911 site were as follows: Forward primer, CGTTCATGTTCCGCCAGGAG and reverse primer, CCGTTCCAGCTCGCGTT $\left(\mathrm{Tm}, 60^{\circ} \mathrm{C}\right)$.

Haploid analysis and SNP-SNP interaction analysis. Haploid analysis of the ADRA2A gene at rs1800035, rs201376588 and rs775887911 was performed using the Haploview 4.2 software $(14,15)$. The degree of linkage is defined by the $\mathrm{R} 2$ value, presented as $0 \leq \mathrm{R} 2 \leq 1$; the larger $\mathrm{R} 2$, the higher the linkage degree, the smaller R2, the lower the linkage degree. SNP-SNP interaction analysis was performed using multi-factor dimensionality reduction (MDR) (16). In the Fruchterman Rheingold diagram, 'n \%' between different SNP sites was used to correlate interaction intensity of between the two factors; the larger the value, the stronger the interaction between these two SNP sites. The smaller the distance within the tree diagram, the stronger the interaction between SNP sites. In contrast, the larger the distance, the weaker the interaction between the SNPs.

Statistical analysis. Statistical analysis was performed using SPSS 20.0 (IBM Corp.). The categorical data are expressed as (n (\%)), Pearson's $\chi^{2}$ test was used for statistical analysis of categorical variables. Continuous data are presented as mean \pm standard deviation. The Kruskal-Wallis test was used for statistical analysis unless otherwise stated. One-way ANOVA was performed to evaluate the difference between the three genotypes group followed by Tukey's post hoc tests. Kruskal-Wallis test was used to analyze the comparison of three discontinuous categorical variables. $\mathrm{P}<0.05$ was considered to indicate a statistically significant difference.

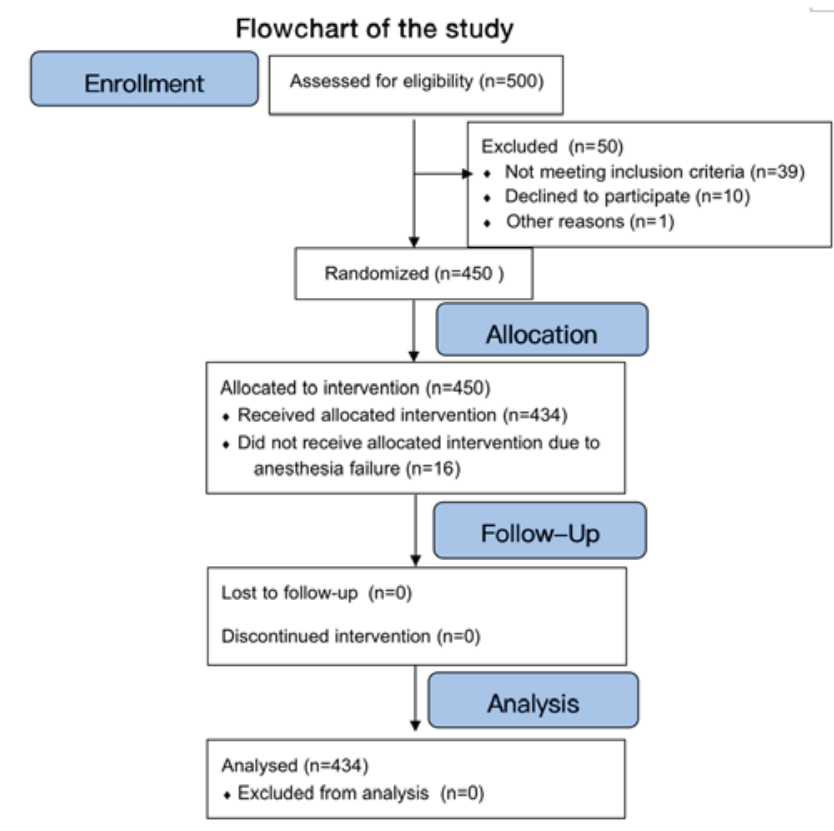

Figure 1. Flowchart demonstrating the selection process for randomized patients.

\section{Results}

Demographic characteristics. A total of 434 Chinese Han women with 434 cases were eventually included in the study (Fig. 1), aged 20-36 years, with a mean age 29.5 3.9 years, including the following: 315 cases with a wild type (CC) ADRA2A rs1800035 locus, accounting for $72.6 \%$, and 119 cases of mutants $(\mathrm{CG}+\mathrm{GG})$ of the ADRA2A rs1800035 locus, accounting for $27.4 \%$; 304 cases of a wild type (CC) ADRA2A rs201376588 locus, accounting for $70.0 \%$, and 130 cases of mutants (CT+TT) of the ADRA2A gene rs201376588 locus, accounting for $30.0 \%$; 310 cases of a wild type (CC) ADRA2A gene rs775887911 locus, accounting for $71.4 \%$, and 124 cases of mutants (CT+TT) of the ADRA2A gene rs775887911 locus, accounting for $28.6 \%$. The demographic characteristics of the subjects are shown in Table I. The three SNP loci of the ADRA2A showed no significant difference in age, BMI, gestational age or cesarean section operation time $(\mathrm{P}>0.05)$.

Association between SNPs, PTh and PTTh. Results of the PTh and PTTh of subjects with different genotypes of the ADRA2A at rs1800035, rs201376588 and rs775887911 loci, at different administration times, are shown in Table II. There was no significant difference between PTh and PTTh for each SNP locus before anesthesia $(\mathrm{P}>0.05)$. After anesthesia, one-way ANOVA results showed that the PTh and PTTh of all SNP site wild type caesarean section puerperae were higher than those of the mutant cesarean section puerperae. This was found to be statistically significant $(\mathrm{P}<0.05)$.

Hemodynamic changes in women during anesthesia for cesarean section. Changes in the hemodynamic indexes of the women during the anesthesia are shown in Fig. 3. After anesthesia, the MAP of the women showed a downward trend, 
A $\dot{C} \dot{A} \dot{G} \dot{G} \dot{C} \dot{C} \dot{C} \ddot{A} \dot{A} \dot{C} \dot{G} \dot{G} \ddot{T} \dot{C} \dot{T} \dot{G} \dot{G} \dot{G} \dot{C}$ rs 1800035 CC

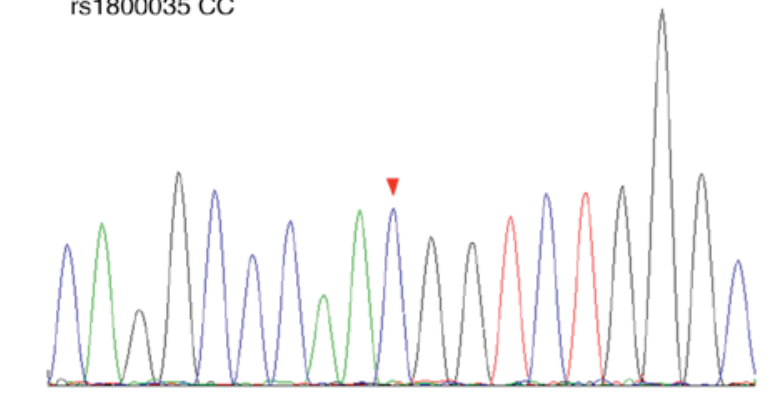

$C \dot{C} \dot{A} \dot{G} \dot{G} \dot{C} \dot{C} \dot{C} \dot{A} \dot{A} \dot{G} \dot{G} \dot{G} \dot{T} \dot{C} \dot{T} \dot{G} \dot{G} \dot{G} \dot{C}$ rs $1800035 \mathrm{GG}$

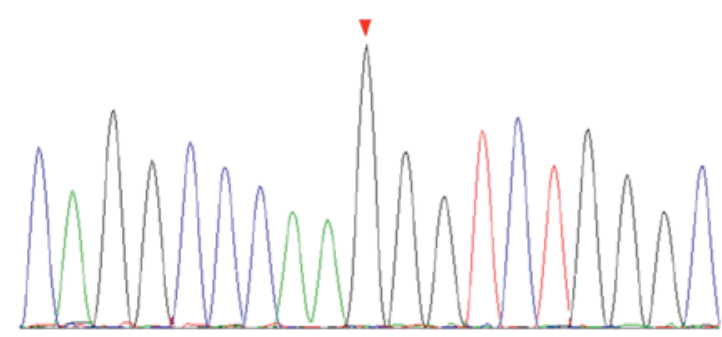
E $\underset{\text { rs } 201376588 \text { CT }}{\dot{T}} \dot{C} \dot{A} \dot{T} \dot{T} \dot{T} \dot{C} \quad \dot{T} \dot{G} \dot{C} \dot{C} \dot{A} \dot{G} \dot{G} \dot{A} \dot{G} \dot{C}$

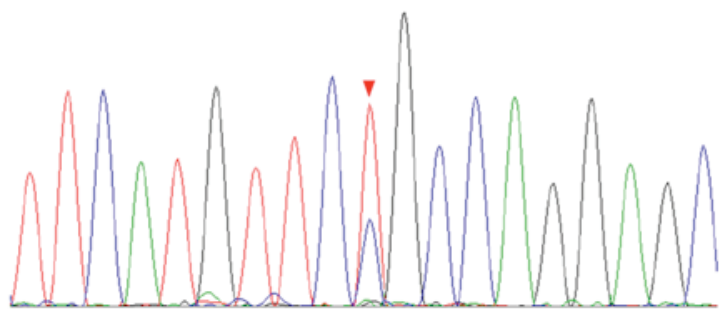

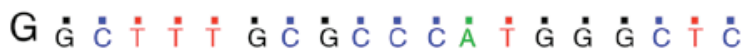
rs775887911 CC

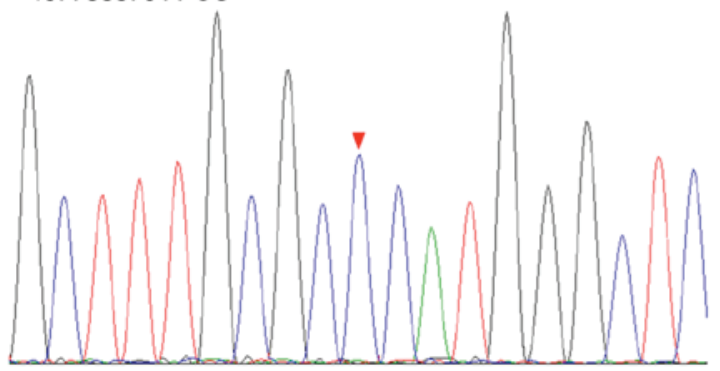

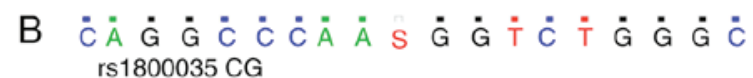

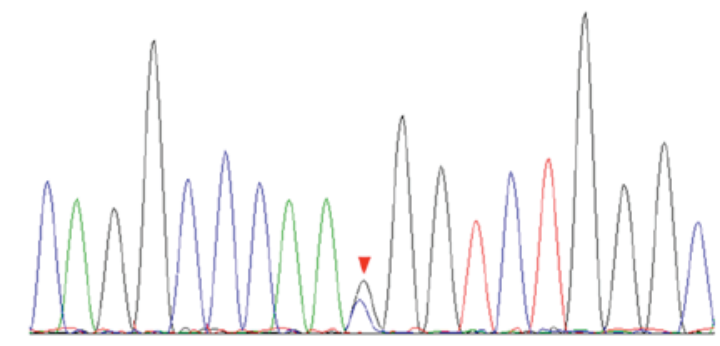

D $\dot{T} \dot{T} \dot{C} \dot{A} \dot{T} \dot{G} \dot{T} \dot{T} \dot{C} \dot{C} \dot{G} \dot{C} \dot{C} \dot{A} \dot{G} \dot{G} \ddot{A} \dot{G} \dot{C}$ rs201376588 CC

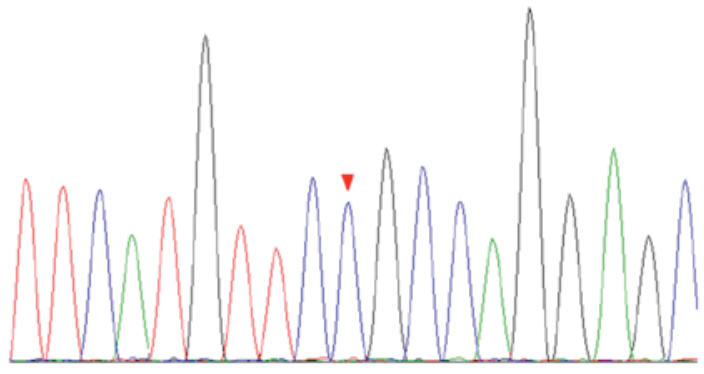

$F \quad \dot{T} \dot{T} \dot{C} \dot{A} \dot{T} \dot{G} \dot{T} \dot{T} \dot{C} \dot{T} \dot{G} \dot{C} \dot{C} \dot{A} \dot{G} \dot{G} \ddot{A} \dot{G}$ rs201376588 CT

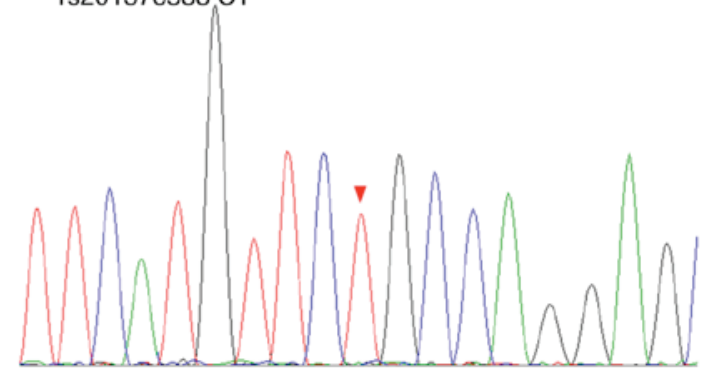

$H \dot{G} \dot{C} \dot{T} \ddot{T} \ddot{T} \dot{G} \dot{C} \dot{G} \dot{C} \dot{C} \dot{C} \ddot{A} \dot{T} \dot{G} \dot{G} \dot{G} \dot{C} \ddot{T} \dot{C}$ rs775887911 CT

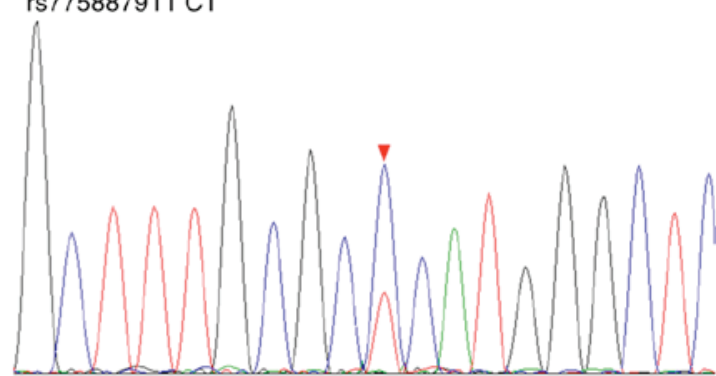

I $\dot{G} \dot{G} \dot{T} \dot{T} \dot{T} \dot{G} \dot{C} \dot{G} \dot{C} \dot{T} \dot{C} A \dot{T} \dot{T} \dot{G} \dot{G} \dot{G} \dot{C} \dot{T}$ rs775887911 TT

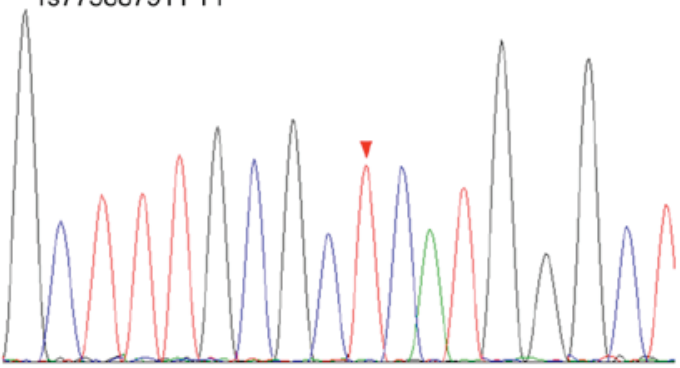

Figure 2. Results of Sanger sequencing of the adrenoceptor $\alpha 2 \mathrm{~A}$ gene. The genotypes of the (A) CC, (B) CG and (C) GG genotypes of the rs1800035 loci. The genotypes of the (D) CC, (E) CT, (F) TT genotypes of the rs201376588 locus. The genotypes of the (G) CC, (H) CT, (I) TT genotypes of the rs 775887911 locus. 
Table I. Demographic characteristic parameters of the subjects.

\begin{tabular}{|c|c|c|c|c|}
\hline SNPs & Age, years & $\mathrm{BMI}, \mathrm{kg} / \mathrm{m}^{2}$ & Gestational age, weeks & Operation time, $\min$ \\
\hline \multicolumn{5}{|l|}{ rs 1800035} \\
\hline $\mathrm{CC}(\mathrm{n}=315)$ & $29.7 \pm 3.2$ & $27.1 \pm 3.4$ & $38.5 \pm 1.9$ & $39.3 \pm 7.2$ \\
\hline $\mathrm{CG}(\mathrm{n}=85)$ & $29.6 \pm 3.7$ & $27.1 \pm 3.6$ & $38.7 \pm 1.8$ & $39.5 \pm 6.7$ \\
\hline GG $(n=34)$ & $30.2 \pm 4.1$ & $27.4 \pm 3.7$ & $39.1 \pm 1.1$ & $40.2 \pm 7.6$ \\
\hline P-value & 0.69 & 0.94 & 0.95 & 0.71 \\
\hline \multicolumn{5}{|l|}{ rs201376588 } \\
\hline $\mathrm{CC}(\mathrm{n}=304)$ & $29.5 \pm 3.5$ & $27.5 \pm 3.6$ & $38.6 \pm 1.8$ & $39.5 \pm 6.8$ \\
\hline CT $(n=87)$ & $29.8 \pm 3.8$ & $27.3 \pm 3.4$ & $38.9 \pm 1.7$ & $39.8 \pm 7.4$ \\
\hline TT $(n=43)$ & $30.1 \pm 3.9$ & $27.6 \pm 3.2$ & $39.2 \pm 1.0$ & $40.3 \pm 7.8$ \\
\hline P-value & 0.85 & 0.93 & 0.77 & 0.90 \\
\hline \multicolumn{5}{|l|}{ rs775887911 } \\
\hline $\mathrm{CC}(\mathrm{n}=310)$ & $28.9 \pm 3.5$ & $27.4 \pm 3.4$ & $38.6 \pm 1.9$ & $39.6 \pm 6.5$ \\
\hline $\mathrm{CT}(\mathrm{n}=88)$ & $29.7 \pm 3.8$ & $27.6 \pm 3.4$ & $38.8 \pm 1.0$ & $39.4 \pm 7.4$ \\
\hline TT $(n=36)$ & $30.3 \pm 4.3$ & $27.4 \pm 3.8$ & $39.1 \pm 1.2$ & $39.9 \pm 8.1$ \\
\hline P-value & 0.66 & 0.76 & 0.80 & 0.75 \\
\hline
\end{tabular}

The data representation here is mean $\pm \mathrm{SD}$. BMI, body mass index; SNP, single-nucleotide polymorphism.

Table II. Comparison of pain threshold and pain tolerance threshold at different time points.

\begin{tabular}{|c|c|c|c|c|}
\hline \multirow[b]{2}{*}{ SNPs } & \multicolumn{2}{|c|}{ PTh, mA } & \multicolumn{2}{|c|}{ PTTh, mA } \\
\hline & Pre-anesthesia & Post-anesthesia & Pre-anesthesia & Post-anesthesia \\
\hline \multicolumn{5}{|l|}{ rs 1800035} \\
\hline $\mathrm{CC}(\mathrm{n}=315)$ & $1.5 \pm 0.4$ & $1.7 \pm 0.5$ & $2.3 \pm 0.5$ & $2.7 \pm 0.5$ \\
\hline $\mathrm{CG}(\mathrm{n}=85)$ & $1.5 \pm 0.5$ & $1.6 \pm 0.4$ & $2.4 \pm 0.4$ & $2.5 \pm 0.6$ \\
\hline $\mathrm{GG}(\mathrm{n}=34)$ & $1.5 \pm 0.4$ & $1.4 \pm 0.6$ & $2.3 \pm 0.6$ & $2.4 \pm 0.4$ \\
\hline P-value & 0.82 & $<0.01$ & 0.78 & 0.02 \\
\hline \multicolumn{5}{|l|}{ rs201376588 } \\
\hline $\mathrm{CC}(\mathrm{n}=304)$ & $1.5 \pm 0.5$ & $1.7 \pm 0.6$ & $2.3 \pm 0.5$ & $2.7 \pm 0.4$ \\
\hline $\mathrm{CT}(\mathrm{n}=87)$ & $1.5 \pm 0.4$ & $1.5 \pm 0.4$ & $2.4 \pm 0.4$ & $2.5 \pm 0.3$ \\
\hline TT $(n=43)$ & $1.5 \pm 0.6$ & $1.5 \pm 0.5$ & $2.4 \pm 0.5$ & $2.4 \pm 0.4$ \\
\hline P-value ${ }^{a}$ & 0.87 & 0.02 & 0.75 & 0.02 \\
\hline \multicolumn{5}{|l|}{ rs775887911 } \\
\hline$C C(n=310)$ & $1.5 \pm 0.5$ & $1.7 \pm 0.5$ & $2.4 \pm 0.4$ & $2.7 \pm 0.3$ \\
\hline $\mathrm{CT}(\mathrm{n}=88)$ & $1.5 \pm 0.6$ & $1.6 \pm 0.5$ & $2.4 \pm 0.3$ & $2.5 \pm 0.4$ \\
\hline $\mathrm{TT}(\mathrm{n}=36)$ & $1.5 \pm 0.4$ & $1.4 \pm 0.6$ & $2.3 \pm 0.4$ & $2.4 \pm 0.5$ \\
\hline P-value ${ }^{a}$ & 0.90 & 0.01 & 0.54 & 0.01 \\
\hline
\end{tabular}

aOne-way ANOVA was used evaluate the difference between the three genotypes group followed by Tukey's post hoc tests. The data representation here is mean \pm SD. PTh, pain threshold; PTTh, pain tolerance threshold; SNP, single-nucleotide polymorphism.

and the HR showed an upward trend. The MAP and HR levels at different time points were statistically significant, as analyzed by one-way ANOVA $(\mathrm{P}<0.05)$. The changes in $\mathrm{SpO}_{2}$ level before and after anesthesia were not significant $(\mathrm{P}>0.05)$. There was no significant difference in MAP, HR or $\mathrm{SpO}_{2}$ between different genotypes at the same time point after anesthesia $(\mathrm{P}>0.05)$.
VAS and Ramsay scores. Results of VAS and Ramsay scores at $6,12,24$ and $48 \mathrm{~h}$ after the operation are shown in Fig. 4. The VAS scores of the puerperae tended to increase at first and then decrease, with the highest level observed at $24 \mathrm{~h}$ after the operation, followed by a decrease at $48 \mathrm{~h}$. The Ramsay score of the puerperae also showed a trend of first increasing and decreasing, reaching a peak at $12 \mathrm{~h}$ and decreasing for the 

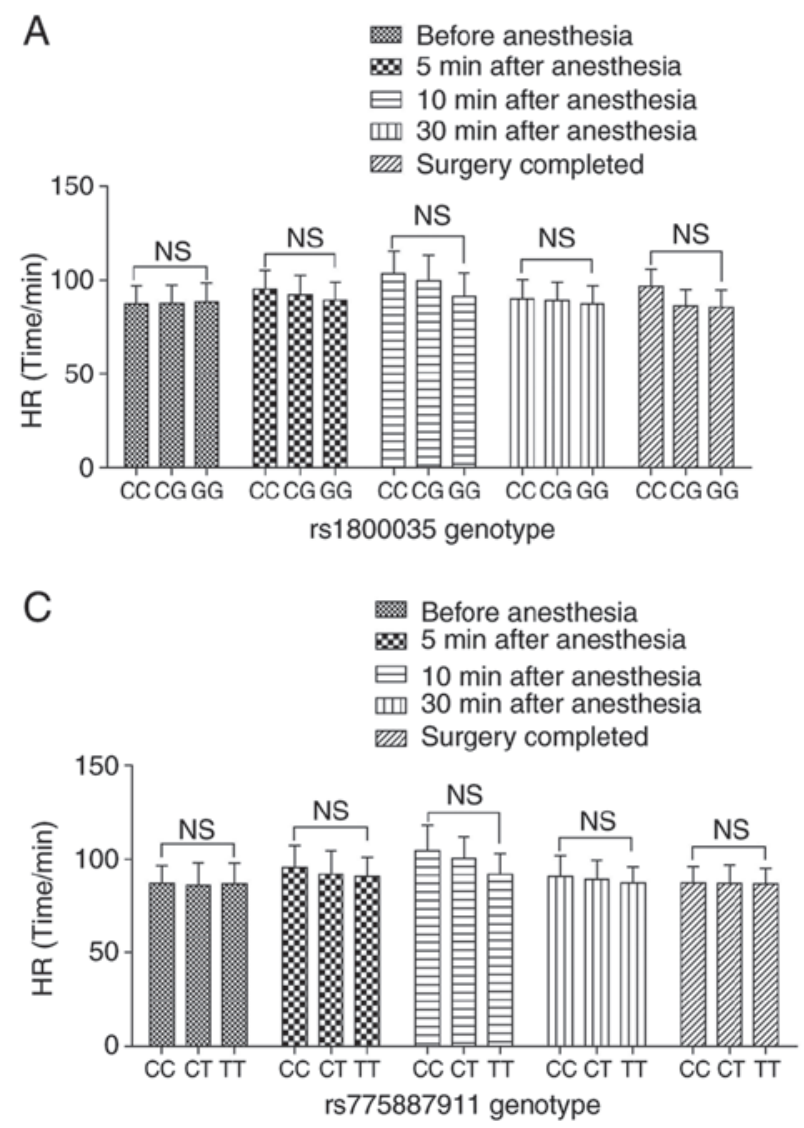

$E$

질 Before anesthesia

$\otimes 5$ min after anesthesia

曰 $10 \mathrm{~min}$ after anesthesia

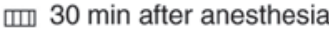

एक्ष Surgery completed

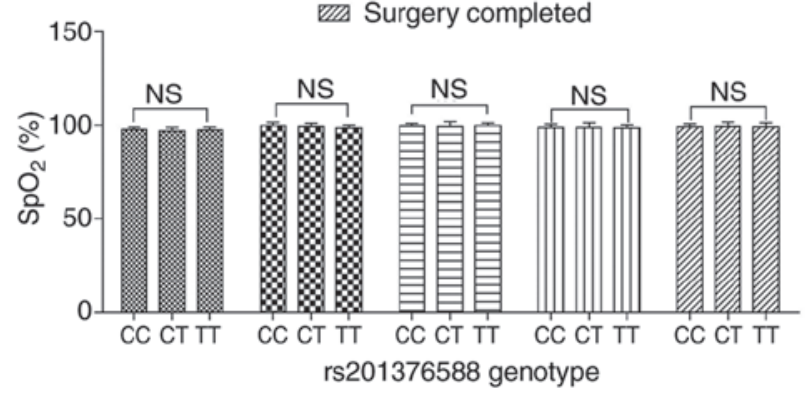

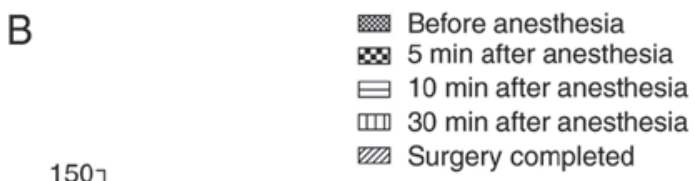
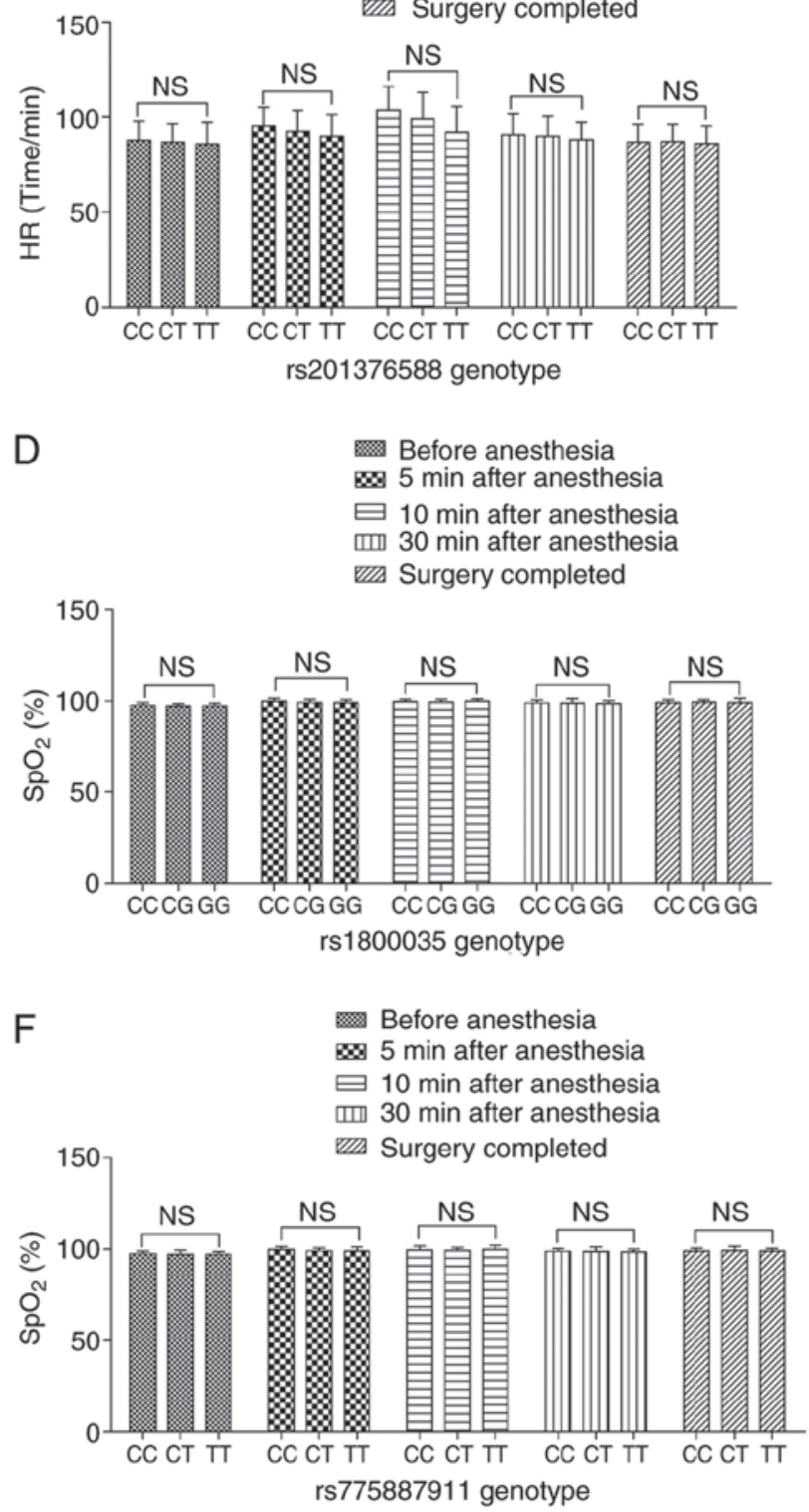

Figure 3. Changes in hemodynamic indexes of the different genotypes during cesarean section. HR scores for the (A) rs1800035, (B) rs210376588, and (C) rs775887911 SNP genotypes. $\mathrm{SPO}_{2}$ values for the (D) rs1800035, (E) rs210376588, and (F) rs775887911 SNP genotypes. HR, heart rate; NS, not significant; SNP, single-nucleotide polymorphism; $\mathrm{SpO}_{2}$, blood oxygen saturation.

remainder of the observation period. The results of comparative analysis of the genotypes of SNPs showed that the VAS score of wild-type puerperae was lower than that of puerperae with mutations at the same time $(\mathrm{P}<0.05)$. However, there was a significant difference in Ramsay score among all genotypes of the puerperae $12 \mathrm{~h}$ after the operation, and the Ramsay score of the wild-type (rs1800035 genotype: CC; rs201376588 genotype: CC; rs775887911 genotype: CC) puerperae was significantly higher compared with that of the mutant genotypes (rs1800035 genotype: CG/GG; rs201376588 genotype: CC/TT; rs775887911 genotype: CT/TT; $\mathrm{P}<0.05$ ).

Comparison of stress response index. Puerperae postoperative Cor and Glu levels at 6, 12, 24, and $48 \mathrm{~h}$ are shown in Fig. 5.
The postoperative levels of Cor and Glu were higher than that before the operation, and the difference was statistically significant $(\mathrm{P}<0.05)$. At 12,24 and $48 \mathrm{~h}$ after the operation, there was a significant difference between the Cor and Glu levels for different genotypes of the rs1800035, rs201376588 and rs775887911 ADRA2A loci. Mothers with mutations had higher levels of Cor and Glu than wild-type mothers $(\mathrm{P}<0.05)$.

Investigation of surgical satisfaction. Results of the investigation into the satisfaction of puerperae with cesarean section are shown in Table III. The satisfaction degree of puerperae after cesarean section was $88.9 \%$ (386/434), of which 204 puerperae were very satisfied (47\%), 182 were satisfied (41.9\%), and 4 were not satisfied $(0.9 \%)$. The comparison of the genotypic 
A

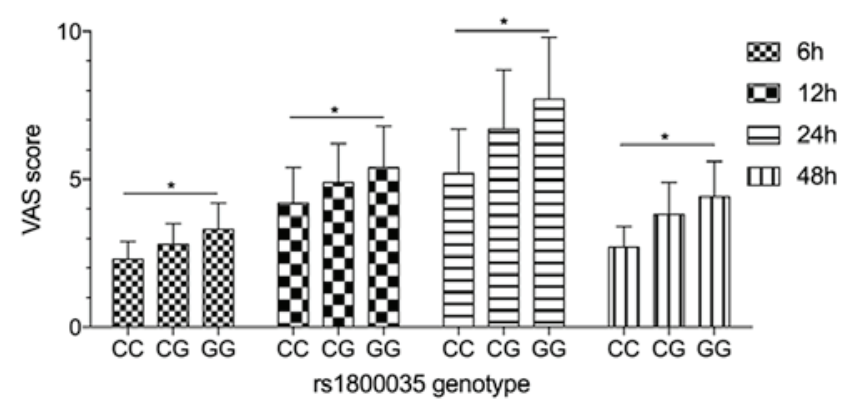

C

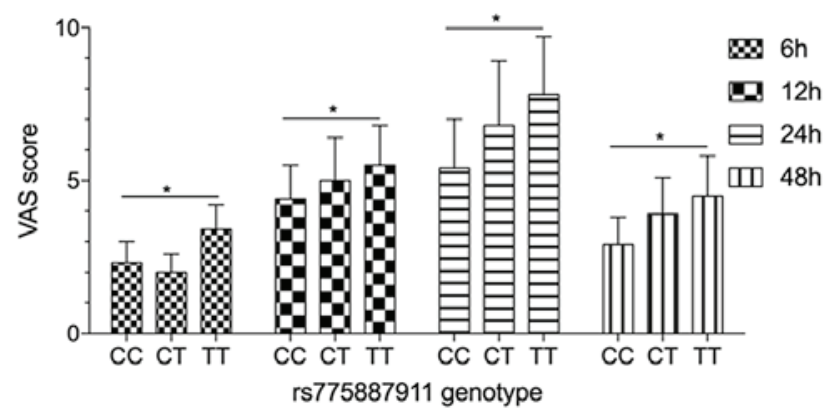

E

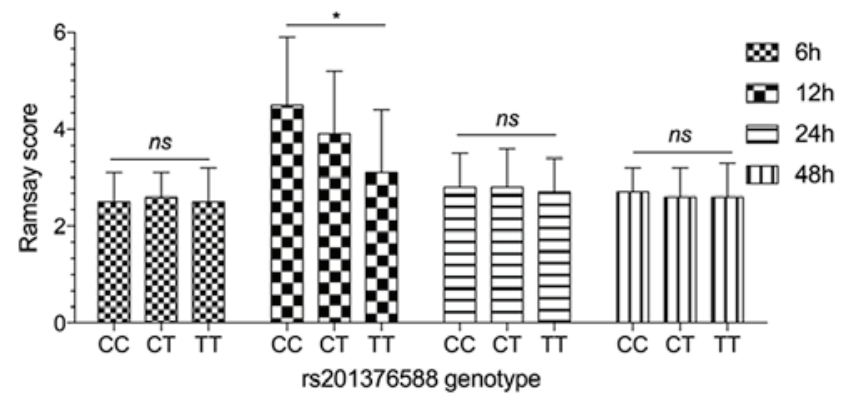

B

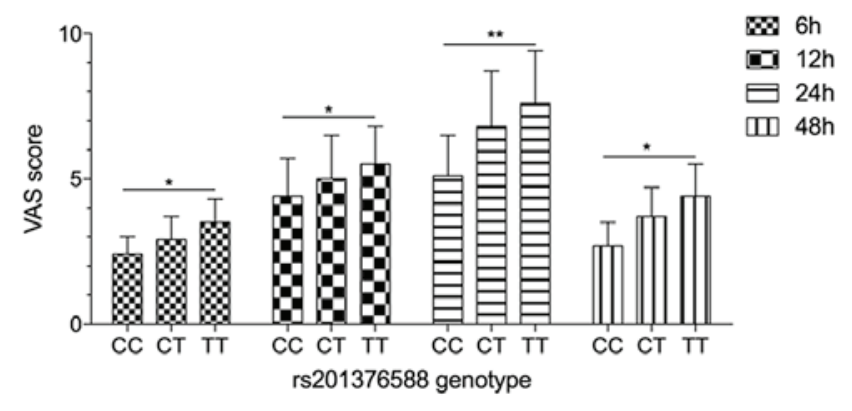

D

W 6 h

[II $12 \mathrm{~h}$ E $24 \mathrm{~h}$

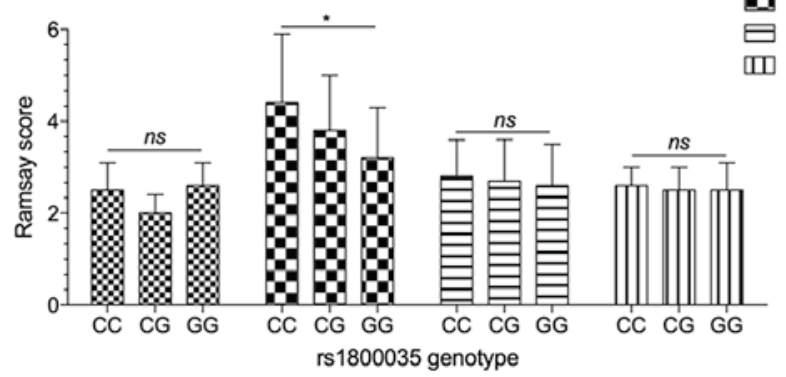

$\mathbf{F}$

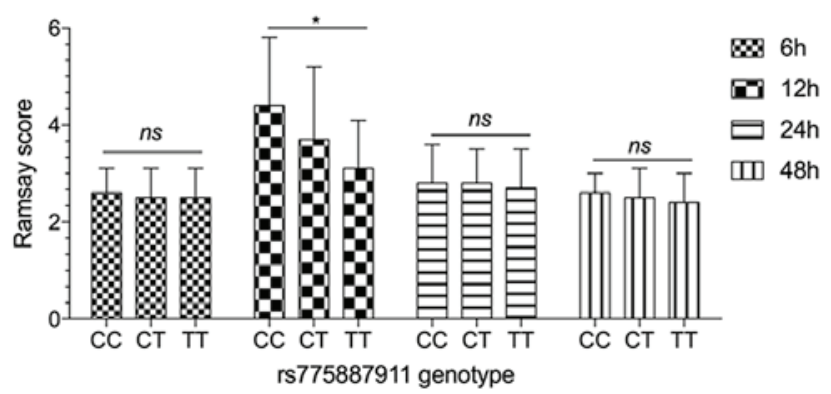

Figure 4. VAS scores and Ramsay sedation scores at 6, 12, 24 and $48 \mathrm{~h}$ post-surgery for the different genotypes of puerperae. VAS scores in the (A) rs1800035, (B) rs210376588 and (C) rs775887911 SNO genotypes. Ramsay sedation scores in the (D) rs1800035, (E) rs210376588 and (F) rs775887911 SNP genotypes. ${ }^{*} \mathrm{P}<0.05,{ }^{* *} \mathrm{P}<0.01$. SNP, single-nucleotide polymorphism; VAS, visual analogue score; NS, no significant difference.

puerperae showed that the satisfaction of the wild type puerperae was higher than the mutant puerperae $(\mathrm{P}<0.05)$.

Postoperative adverse reaction. Adverse reactions after cesarean section in this study included nausea (30 cases), vomiting ( 22 cases), chills ( 31 cases) and pruritus (12 cases), as shown in Table IV. Kruskal-Wallis test showed that there was no significant difference in postoperative adverse reactions between the different genotypes $(\mathrm{P}>0.05)$.

Linkage disequilibrium analysis of rs1800035, rs201376588 and rs775887911 loci of the ADRA2A. Linkage disequilibrium of the rs1800035, rs201376588 and rs775887911 loci of the ADRA2A was analyzed by Haploview 4.2, and the results showed that $\mathrm{r}^{2}=0.2$ (Fig. 6). In addition, multifactorial dimensionality reduction (MDR) was used to analyze the interaction of rs1800035, rs201376588 and rs775887911 loci of the ADRA2A. The results showed that there was a strong interaction between rs1800035 and rs775887911 loci (Fig. 7).

\section{Discussion}

The present study analyzed the effects of ADRA2A rs1800035, rs201376588 and rs775887911 SNPs on dexmedetomidine anesthesia and analgesia in Chinese Han women. The results showed that the mutations of rs1800035, rs201376588 and rs775887911 in the ADRA2A reduced the anesthetic and analgesic effect of dexmedetomidine after cesarean section in women of the Chinese Han dynasty. The postoperative analgesia of mutant maternal women may require more doses of analgesic drugs, but the mutation did not affect the safety of the medication.

The pain after cesarean section is most severe at $48 \mathrm{~h}$ post-surgery, and is mainly somatic pain caused by the abdominal surgery incision and uterine contraction pain. The two pain pathways in the spinal cord and above are different (17); the pain caused by uterine contraction is visceral pain, mainly transmitted by the sympathetic afferent fibers into the cervix and lower abdomen through the plexus 
A
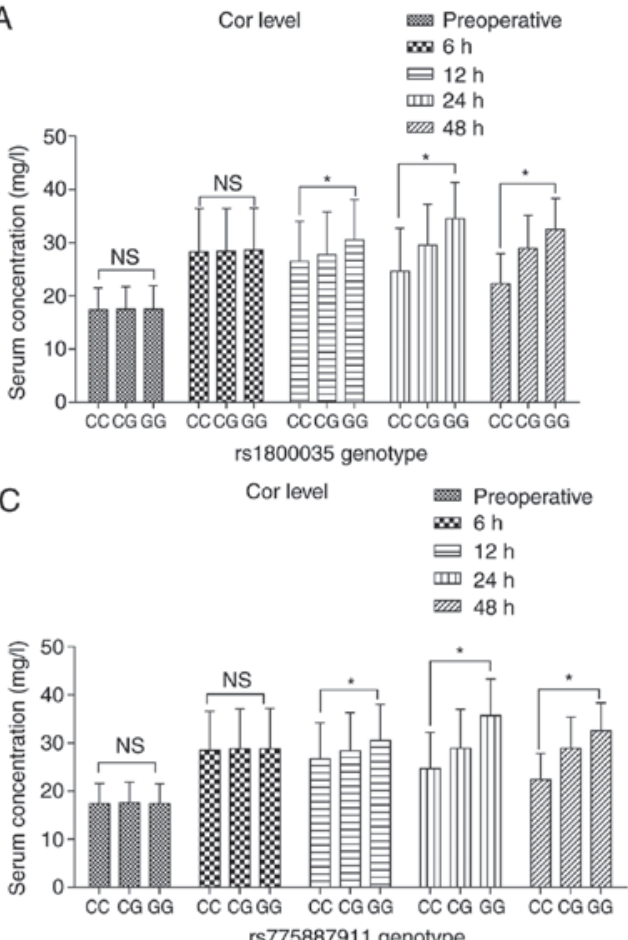

E

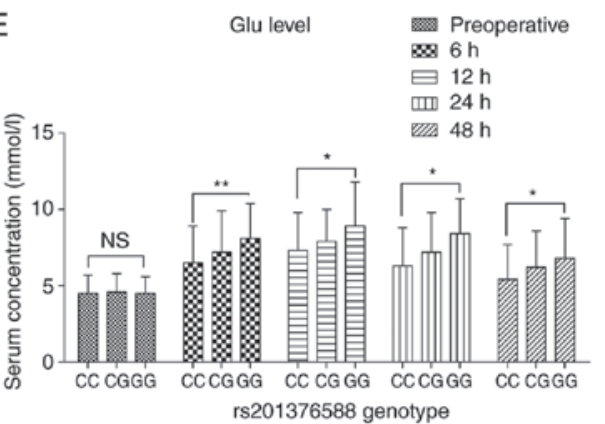

B
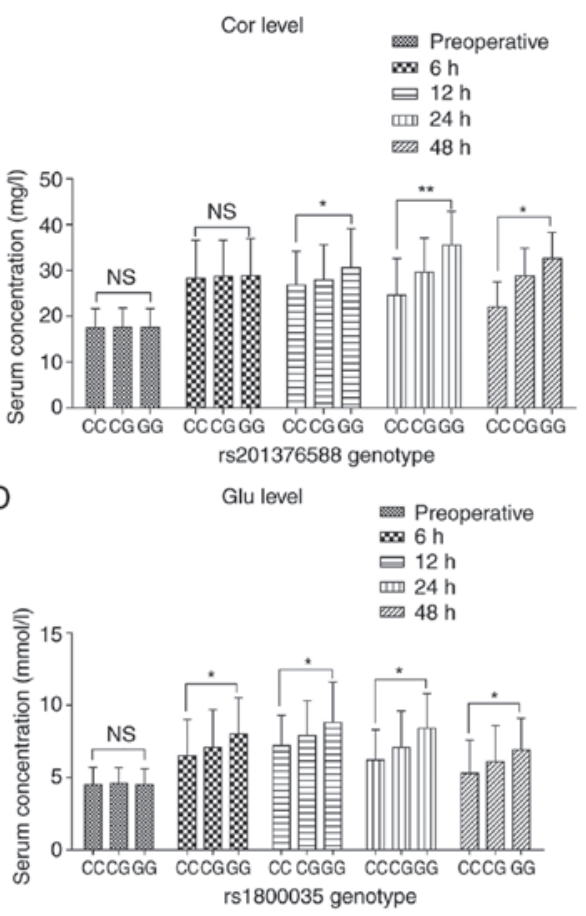

F

Glu level

픔 Preoperative

$\infty 6 \mathrm{~h}$

띤 $24 \mathrm{~h}$

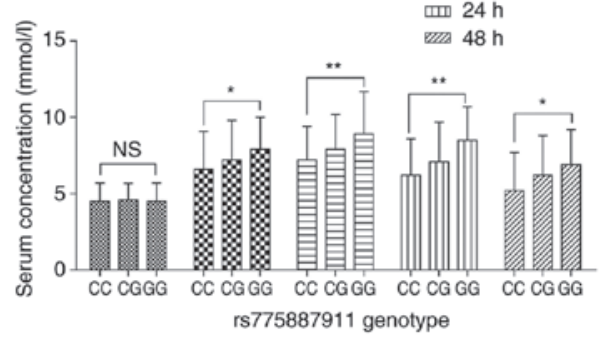

Figure 5. Serum Cor and Glu content in different genotypes of puerperae. Cor scores in the (A) rs1800035, (B) rs210376588 and (C) rs775887911 SNP genotypes. Glu scores in the (D) rs1800035, (E) rs210376588 and (F) rs775887911 SNP genotypes. * $\mathrm{P}<0.05,{ }^{* *} \mathrm{P}<0.01$. SNP, single-nucleotide polymorphism; NS, no significant difference; Cor, cortisol; Glu, glucose.

into the lumbar sympathetic chain and then into the spinal dorsal horn via the T10, 11, 12 and L1 segments (18); the pain in the abdominal incision is located at the incision and passes into the spinal dorsal horn through the spinal nerve of the T10-L1 segment (19). Pain after cesarean section will lead to maternal sympathetic excitement and increased secretion of catecholamines and metabolic hormones, thereby affecting the maternal digestive system function and physical recovery; this is a main reason for the negative emotions exhibited by new mothers, affecting their lactation $(20,21)$. Morphine is a classical epidural analgesic that enters the cerebrospinal fluid and binds to opiate receptors in the glia of the dorsal horn of the spinal cord, producing a potent, long-lasting analgesic effect (22). However, studies have shown that morphine can excite $\mu$ receptors, resulting in nausea, vomiting, pruritus and other adverse reactions (23).

In this study, dexmedetomidine hydrochloride combined with ropivacaine was used for continuous epidural auto-control analgesia after cesarean section surgery. Dexmedetomidine hydrochloride is a new type of highly selective $\alpha 2-\mathrm{AR}$ agonist.
Its main mechanism is that it acts on presynaptic $\alpha 2$-ARs of spinal cord $\mathrm{C}$ class or $\mathrm{A} \delta$ nerve fibers, reducing the release of nerve fiber neurotransmitters, and thus nociceptive signals cannot be transmitted between neurons, thereby achieving the effect of analgesia (24). At the same time, dexmedetomidine can also act on the postsynaptic membrane $\alpha 2$-ARs in the posterior horn neurons of the spinal cord, causing hyperpolarization of the neurons in the posterior horn of the spinal cord. The nociceptive signals thus cannot be transmitted in the nerve fibers, resulting in antinociceptive effects (25). In addition, dexmedetomidine also has high liposolubility and can be absorbed from the bloodstream through the blood-brain barrier into the human central nervous system locus coeruleus to produce a calming effect, to eliminate anxiety, tension and fear caused by pain (25). Some studies have shown that dexmedetomidine has a synergistic effect with other local anesthetic drugs, which can enhance the analgesic effect and reduce the use of local anesthetic drugs, while dexmedetomidine can also be effective in inhibiting postoperative chills and reducing nausea, vomiting, pruritus and other adverse reactions (26). 
Table III. Comparison of the satisfaction of puerperae with cesarean section by genotype.

\begin{tabular}{|c|c|c|c|c|c|}
\hline SNPs & Very satisfied, n (\%) & Satisfied, n (\%) & Normal, n (\%) & Not satisfied, n (\%) & Satisfaction rate, $\%$ \\
\hline \multicolumn{6}{|l|}{ rs1800035 } \\
\hline $\mathrm{CC}(\mathrm{n}=315)$ & $172(54.6)$ & $126(40.0)$ & $16(5.1)$ & $1(0.3)$ & $94.6 \%$ \\
\hline $\mathrm{CG}(\mathrm{n}=85)$ & $26(30.6)$ & $42(49.4)$ & $16(18.8)$ & $1(1.2)$ & $80.0 \%$ \\
\hline $\mathrm{GG}(\mathrm{n}=34)$ & $6(17.6)$ & $14(41.2)$ & $12(35.3)$ & $2(5.9)$ & $58.8 \%$ \\
\hline P-value ${ }^{a}$ & & & & & $<0.01$ \\
\hline \multicolumn{6}{|l|}{ rs201376588 } \\
\hline $\mathrm{CC}(\mathrm{n}=304)$ & $157(51.6)$ & $121(39.8)$ & $24(7.9)$ & $2(0.7)$ & $91.5 \%$ \\
\hline CT $(n=87)$ & $40(46.0)$ & $32(36.8)$ & $14(16.1)$ & $1(1.1)$ & $82.8 \%$ \\
\hline $\mathrm{TT}(\mathrm{n}=43)$ & $7(16.3)$ & $29(67.4)$ & $6(14.0)$ & $1(2.3)$ & $83.7 \%$ \\
\hline P-value ${ }^{a}$ & & & & & 0.04 \\
\hline \multicolumn{6}{|l|}{ rs775887911 } \\
\hline $\mathrm{CC}(\mathrm{n}=310)$ & $169(54.5)$ & $118(38.1)$ & $21(6.8)$ & $2(0.6)$ & $92.6 \%$ \\
\hline CT $(n=88)$ & $25(28.4)$ & $44(50.0)$ & $18(20.5)$ & $1(1.1)$ & $78.4 \%$ \\
\hline TT $(n=36)$ & $10(27.8)$ & $20(55.6)$ & $5(13.9)$ & $1(2.8)$ & $83.3 \%$ \\
\hline P-value ${ }^{a}$ & & & & & $<0.01$ \\
\hline
\end{tabular}

${ }^{a}$ Kruskal-Wallis test was performed to evaluate the difference between the three genotypes group. SNP, single-nucleotide polymorphism.

Table IV. Comparison of postoperative adverse effects of various genotypic puerperae.

\begin{tabular}{lcccc}
\hline SNPs & Nausea & Vomiting & Chills & Pruritus \\
\hline rs1800035 & & & & \\
CC $(n=315)$ & 23 & 12 & 22 & 10 \\
CG $(n=85)$ & 5 & 8 & 6 & 2 \\
GG $(n=34)$ & 2 & 2 & 3 & 0 \\
P-value & 0.88 & 0.11 & 0.92 & 0.55 \\
rs201376588 & & & & \\
CC $(n=304)$ & 22 & 14 & 23 & 9 \\
CT $(n=87)$ & 5 & 6 & 5 & 3 \\
TT $(n=43)$ & 3 & 2 & 3 & 0 \\
P-value & 0.89 & 0.69 & 0.84 & 0.45 \\
rs775887911 & & & & \\
CC $(n=310)$ & 23 & 13 & 21 & 9 \\
CT $(n=88)$ & 6 & 6 & 6 & 2 \\
TT $(n=36)$ & 1 & 4 & 4 & 1 \\
P-value & 0.45 & 0.29 & 0.85 & 0.92 \\
\hline
\end{tabular}

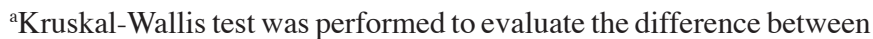
the three genotypes group. SNP, single-nucleotide polymorphism.

However, there are often some differences in the effect of the same dose of the drug in clinical application (27). The existence of variability in drug response between individuals has long been known (28). In drug therapy, susceptibility to toxic or inadequately treated individuals is important. Genetic polymorphisms of genes responsible for drug reactions are expected to be used to track differences between individuals (29). The influence of genetic factors is not clear. After

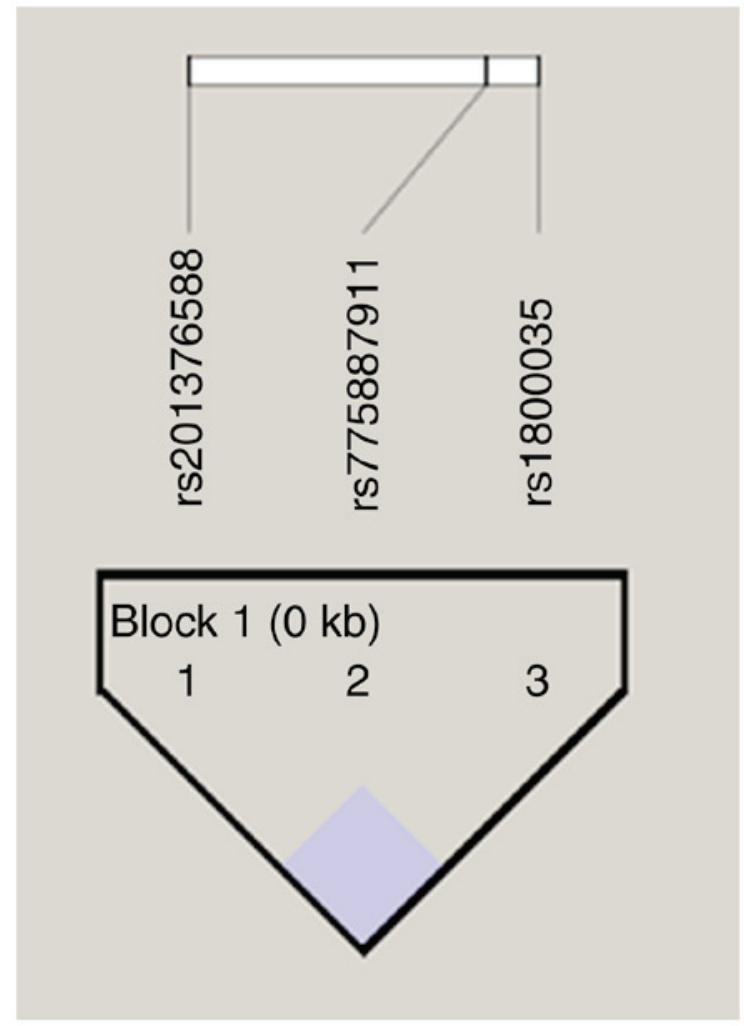

Figure 6. Linkage disequilibrium analysis of rs1800035, rs201376588 and rs775887911 loci of adrenoreceptor $\alpha 2 \mathrm{~A}$.

searching the KEGG database, the target of dexmedetomidine was determined to be the ADRA2A, and the effect of SNPS of this gene on the analgesic effect of dexmedetomidine was analyzed. Due to technical limitations, only three potential SNP loci that affected the structure and function of the $\alpha 2$-AR 

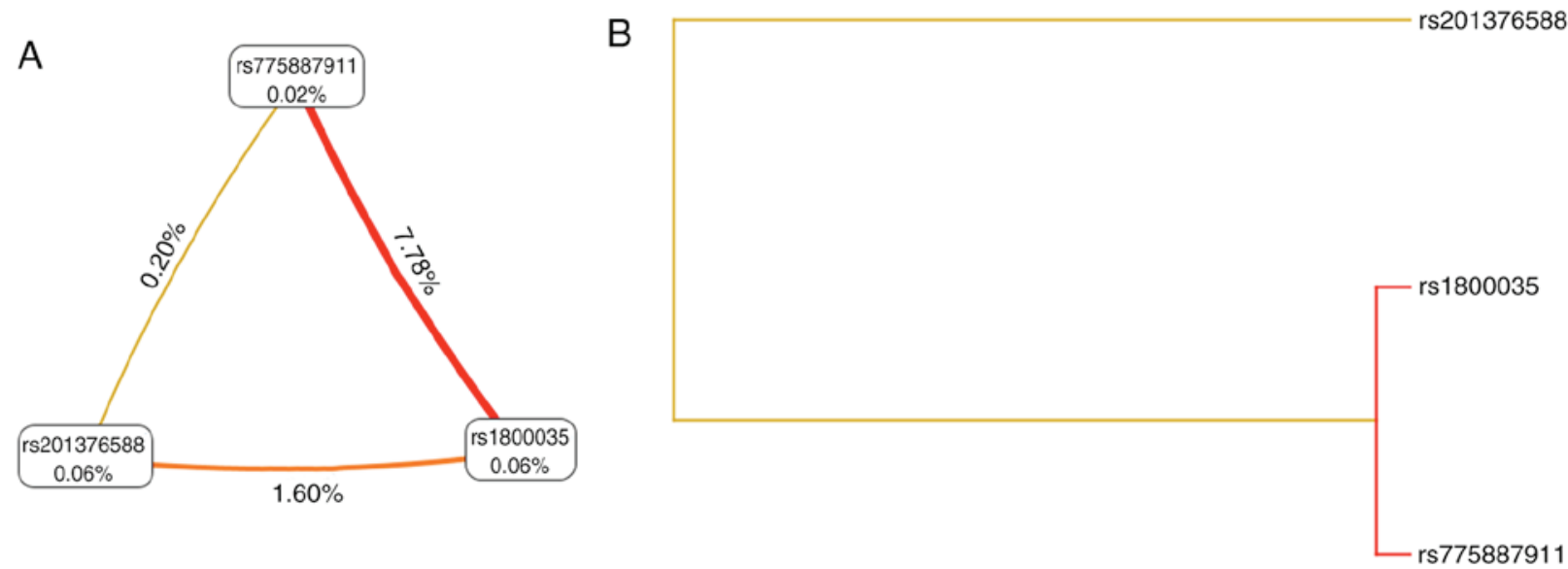

Figure 7. Interaction analysis of the rs1800035, rs201376588 and rs775887911 loci of the adrenoreceptor $\alpha 2 \mathrm{~A}$ gene. (A) Fruchterman-Rheingold diagram representing interactions between the loci. ' $n \%$ ' between different SNP sites indicates the intensity of the interaction between the two factors. A larger 'n\%' indicates a stronger interaction between these two factors. (B) Tree diagram representing the interaction between SNPs. Two adjacent SNP sites demonstrate a strong interaction, and the farther the distance is, the weaker the interaction. SNP, single-nucleotide polymorphism.

protein were screened, namely, rs1800035, rs201376588 and rs775887911.

There were no significant differences for age, gestational age, BMI, operation time and all of the genotypes of SNPs of the subjects enrolled in this study, which precluded the interference of these factors. Nie et al (30) showed that intravenous injections with dexmedetomidine at an initial stage after delivery can increase maternal PTh and PTTh in cesarean section patients, consistent with the results of the present study. The present study compared the PTh and PTTh values of ADRA2A rs1800035, rs201376588 and rs775887911 genotypes at different times after administration of analgesia.

Inflammatory reactions induced by traumatic stimulation after cesarean section can lead to inflammatory mediator release. Without direct analgesia, this can also cause maternal vasodilatation, tissue edema, an increase in the sensitivities of the effector and a decrease in pain threshold, which leads to hyperalgesia (31). From the results of the present study, it can be seen that women who are wild-type at the rs1800035, rs201376588 and rs775887911 loci of the ADRA2A have a higher pain threshold than mutant puerperae. Since obviously mutant puerperae are more sensitive to pain, these types of patients may need higher or additional doses of drugs for anesthesia and analgesia. In addition, the hemodynamic parameters of post-anesthesia puerperae had a downward MAP trend, and their HR showed an upward trend, which may be due to hypotension caused by sympathetic nerve blocks during anesthesia, or possibly due to a slightly higher dose of drug. There was no significant change in the level of $\mathrm{SpO}_{2}$, suggesting that respiratory inhibition was not produced under the dosage of the drug in this study. Similarly, the analysis results of the hemodynamic level between different locus genotypes showed no significant difference in the level of MAP, HR or $\mathrm{SpO}_{2}$ at the same time point after anesthesia, indicating that there was no significant association between the rs1800035, rs201376588, and rs775887911 loci SNPs of the ADRA2A and the level of hemodynamics of the puerperae during the anesthesia. We speculate that the differences between the different genotypes of puerperae may have been reduced by compensatory, rehydration and vasoconstrictor drugs, but this needs to be confirmed.

Dexmedetomidine has a strong postoperative analgesic effect. It can be seen from the results of this study that the VAS score of puerperae first increased and then decreased during the observed timeframe after the operation, with the highest level at $24 \mathrm{~h}$ post-surgery. Our analysis suggests that dexmedetomidine inhibits the transmission of pain information by entering spinal $\alpha 2-A R s$ through spinal pinholes and diffusion. The Ramsay score of puerperae during the observed timeframe after surgery also showed a trend of first rising and then decreasing, with the highest level at $12 \mathrm{~h}$ after the operation, followed by a decline up to the last observation at $48 \mathrm{~h}$. It is hypothesized that dexmedetomidine exerts a sedative effect by the direct action of cerebrospinal fluid on locus coeruleus $\alpha 2$-ARs. A previous study (32) found that the ADRA2A C1291C, C1291G and G1291G sites carrying variant genotypes had higher bispectral index scores and Ramsay sedation scores, indicating a longer time to falling asleep. It has been reported that that the ADRA2A-1291 C-> G polymorphism is associated with abnormal cortisol response to dexamethasone, elevated glucose levels and increased diastolic blood pressure. Pathophysiology may involve a change in the density of $\alpha 2 \mathrm{~A}-\mathrm{ARs}$, which makes the sympathetic-hypothalamic-pituitary-adrenal system unstable in those with genetic susceptibility in the ADRA2A promoter (33). Another study found that the ADRA2A-1291C/G SNP is associated with the efficacy of methylphenidate (MPH) in the treatment of attention deficit/hyperactivity disorder in children and adolescents in Taiwan. Reactive subjects carrying the homozygous-1291G allele are more likely to respond better to MPH therapy, indicating that the $-1191 \mathrm{C} / \mathrm{G}$ SNP is very sensitive to MPH (34). In addition, as seen from the results of the present study, the VAS score of wild-type puerperae was lower than the mutant puerperae at the same time after surgery, while the Ramsay scores of wild-type puerperae $12 \mathrm{~h}$ after surgery were significantly higher than the mutant puerperae, indicating that wild-type puerperae are more sensitive to dexmedetomidine than mutant puerperae, probably because genetic mutations alter the structure and/or function of the 
protein to weaken the interaction between dexmedetomidine and $\alpha 2$-ARs. It is also possible that this SNP site mutation affects protein expression levels. However, there are limited studies on the effects of this SNP site mutation on the structure and function of $\alpha 2$-ARs, and further research is needed. However, according to the results of sequence analysis, the C>G mutation at rs1800035 will cause the Asn amino acid to be mutated into a Lys amino acid, from a neutral amino acid to a basic amino acid. The $\mathrm{C}>\mathrm{T}$ mutation at rs201376588 leads to the mutation of Arg amino acid to Cys, from basic amino acid to neutral amino acid, and the $\mathrm{C}>\mathrm{T}$ mutation at rs775887911 leads to the mutation of Pro amino acid to Leu, both of which are neutral amino acids. These types of mutations have changed the amino acid sequence. It is unclear whether it causes changes in protein structure. However, from the results of the present study, it may be speculated that the changes in these amino acid sequences affect the structure and function of $\alpha 2$-ARs. The postoperative maternal Cor and Glu levels were higher than the preoperative levels, which is also due to the normal physiological trauma caused by surgery. In addition, this study found that the levels of Cor and Glu of the mutant puerperae were higher than that of the wild-type puerperae at the same time after the operation, indicating that the stress response of mutant puerperae is stronger than that of wild-type mothers, and puerperae with a mutation in the locus are more sensitive to the stress reaction caused by surgical trauma. The specific mechanism needs further study. Furthermore, the satisfaction degree of wild-type puerperae with postoperative analgesia was higher than that of mutant puerperae, which also confirms that the mutant puerperae are less sensitive to the dexmedetomidine than the wild type. Fortunately, based on the results of this study, the mutations did not affect the adverse reactions produced by dexmedetomidine.

There are limitations with this study. Due to technical limitations, the concentration and receptor density of dexmedetomidine was not detected in this study. In future studies, we plan to analyze the concentration of dexmedetomidine by means of mass spectrometry. An in vitro study model to study the density of its receptors will be designed to analyze the relationship between ADRA2A polymorphisms on concentrations of dexmedetomidine and receptor densities. In addition, the association between anesthetic analgesic effects and SNPs at different dexmedetomidine doses was not explored, which is also one of the focuses of further research.

It can be concluded from the results of this study that the mutation of the ADRA2A at the rs1800035, rs201376588 and rs775887911 loci can reduce the anesthetic and analgesic effect on Chinese Han cesarean section puerperae; for postoperative analgesia, they may require more anesthetics, but the gene mutations at those SNPs do not affect drug safety.

\section{Acknowledgements}

Not applicable.

\section{Funding}

The present study was supported by grants from Medical Health Science and Technology Program of Zhejiang Province (grant no. 2018KY323).

\section{Availability of data and materials}

The datasets used and/or analyzed during the present study are available from the corresponding author on reasonable request.

\section{Authors' contributions}

ZMF and DDW designed the study. ZMF, BWH, TTM and QDW performed the data collection. ZMF, BWH and TTM analyzed and interpreted the data. ZMF drafted the manuscript and DDW revised the manuscript.

\section{Ethics approval and consent to participate}

This study was approved by the medical ethics committee of Tongde Hospital of Zhejiang Province hospital (Zhejiang, China), and all women provided informed consent.

\section{Patient consent for publication}

Not applicable.

\section{Competing interests}

The authors declare that they have no competing interests.

\section{References}

1. Betran AP, Torloni MR, Zhang JJ and Gülmezoglu AM; WHO Working Group on Caesarean Section: WHO statement on caesarean section rates. BJOG 123: 667-670, 2016.

2. Liu Xiao Jing JG and Yan Y: Analysis of cesarean section rate and indication of cesarean section in 10 years. Maternal Child Health Care China 30: 551-553, 2015.

3. Snijder CA, Cornette JM, Hop WC, Kruip MJ and Duvekot JJ: Thrombophylaxis and bleeding complications after cesarean section. Acta Obstet Gynecol Scand 91: 560-565, 2012.

4. Blaudszun G, Lysakowski C, Elia N and Tramèr MR: Effect of perioperative systemic $\alpha 2$ agonists on postoperative morphine consumption and pain intensity: Systematic review and metaanalysis of randomized controlled trials. Anesthesiology 116: 1312-1322, 2012

5. Unlugenc $\mathrm{H}$, Gunduz M, Guler T, Yagmur $\mathrm{O}$ and Isik G: The effect of pre-anaesthetic administration of intravenous dexmedetomidine on postoperative pain in patients receiving patient-controlled morphine. Eur J Anaesthesiol 22: 386-391, 2005.

6. Qi X, Chen D, Li G, Huang X, Li Y, Wang X and Li Y: Comparison of intrathecal dexmedetomidine with morphine as adjuvants in cesarean sections. Biol Pharm Bull 39: 1455-1460, 2016.

7. Pettersson FD, Grönbladh A, Nyberg F, Sundström-Poromaa I and Åkerud H: The A118G single-nucleotide polymorphism of human $\mu$-opioid receptor gene and use of labor analgesia. Reprod Sci 19: 962-967, 2012.

8. Tian WN, Duzic E, Lanier SM and Deth RC: Determinants of alpha 2-adrenergic receptor activation of $\mathrm{G}$ proteins: Evidence for a precoupled receptor/G protein state. Mol Pharmacol 45: 524-531, 1994.

9. Kohli U, Muszkat M, Sofowora GG, Harris PA, Friedman EA, Dupont WD, Scheinin M, Wood AJ, Stein CM and Kurnik D: Effects of variation in the human alpha2A- and alpha2C-adrenoceptor genes on cognitive tasks and pain perception. Eur J Pain 14: 154-159, 2010.

10. Farshchi A and Ghiasi G: Comparison the analgesic effects of single dose administration of tramadol or piroxicam on postoperative pain after cesarean delivery. Acta Med Iran 48: 148-153, 2010.

11. Kelly AM: The minimum clinically significant difference in visual analogue scale pain score does not differ with severity of pain. Emerg Med J 18: 205-207, 2001.

12. Rasheed AM, Amirah MF, Abdallah M, P J P, Issa M and Alharthy A: Ramsay sedation scale and richmond agitation sedation scale: A Cross-sectional study. Dimens Crit Care Nurs 38: 90-95, 2019. 
13. Camilleri M, Lembo A and Katzka DA: Opioids in gastroenterology: Treating adverse effects and creating therapeutic benefits. Clin Gastroenterol Hepatol 15: 1338-1349, 2017.

14. Chon J, Hong JH, Kim J, Han YJ, Lee BW, Kim SC, Kim DH, Yoo SD, Kim HS and Yun DH: Association between BH3 interacting domain death agonist (BID) gene polymorphism and ossification of the posterior longitudinal ligament in Korean population. Mol Biol Rep 41: 895-899, 2014.

15. Lv Y, Jia C, Jiang A, Zhang H, Wang Y, Liu F, Yang L, Sun Y, Lv R and Song X: Analysis of association between MGMT and p53 gene single nucleotide polymorphisms and laryngeal cancer. Anticancer Res 37: 4399-4403, 2017.

16. Yang $\mathrm{CH}$, Chuang LY and Lin YD: Multiobjective differential evolution-based multifactor dimensionality reduction for detecting gene-gene interactions. Sci Rep 7: 12869, 2017.

17. Lavand'homme P: Postcesarean analgesia: Effective strategies and association with chronic pain. Curr Opin Anaesthesiol 19: 244-248, 2006

18. Zwissler B: Regional anesthesia and analgesia for labor and delivery. N Engl J Med 348: 1818-1820, 2003.

19. McDonnell JG, O'Donnell BD, Farrell T, Gough N, Tuite D, Power C and Laffey JG: Transversus abdominis plane block: A cadaveric and radiological evaluation. Reg Anesth Pain Med 32: 399-404, 2007.

20. Niklasson B, Georgsson Öhman S, Segerdahl M and Blanck A: Risk factors for persistent pain and its influence on maternal wellbeing after cesarean section. Acta Obstet Gynecol Scand 94 622-628, 2015

21. Borges NC, Pereira LV, de Moura LA, Silva TC and Pedroso CF Predictors for moderate to severe acute postoperative pain after cesarean section. Pain Res Manag 2016: 5783817, 2016.

22. Stavropoulou E, Balkamou XA, Giannaka F, Papadopoulos G, Papadopoulos K and Stamatopoulos G: 303: Opiod-induced adverse reactions of intravenous patient controlled analgesia: Comparison of morphine and fentanyl for acute postoperative analgesia. Regional Anesthesia Pain Med 33 (Suppl 1): e166, 2008.

23. Chan K, Brodsky M, Davis T, Franklin S, Inturrisi CE and Yoburn BC: The effect of the irreversible mu-opioid receptor antagonist clocinnamox on morphine potency, receptor binding and receptor mRNA. Eur J Pharmacol 287: 135-143, 1995.

24. Dawson C, Ma D, Chow A and Maze M: Dexmedetomidine enhances analgesic action of nitrous oxide: Mechanisms of action. Anesthesiology 100: 894-904, 2004.
25. Afonso J and Reis F: Dexmedetomidine: Current role in anesthesia and intensive care. Rev Bras Anestesiol 62: 118-133, 2012.

26. Ren C, Chi M, Zhang Y, Zhang Z, Qi F and Liu Z Dexmedetomidine in postoperative analgesia in patients undergoing hysterectomy: A CONSORT-prospective, randomized, controlled trial. Medicine (Baltimore) 94: e1348, 2015.

27. Marto N, Morello J, Monteiro EC and Pereira SA: Implications of sulfotransferase activity in interindividual variability in drug response: Clinical perspective on current knowledge. Drug Metab Rev 49: 357-371, 2017.

28. Lim MH, Kim JW, Song EY, Kim TH, Park TW, Lee HJ, Paik KC and Kim HW: COMT gene polymorphism association and drug response in Tourette syndrome. Psychiatr Genet 19: 158, 2009.

29. Zastrozhin MS, Brodyansky VM, Skryabin VY, Grishina EA, Ivashchenko DV, Ryzhikova KA, Savchenko LM, Kibitov AO, Bryun EA and Sychev DA: Pharmacodynamic genetic polymorphisms affect adverse drug reactions of haloperidol in patients with alcohol-use disorder. Pharmgenomics Pers Med 10: 209-215, 2017.

30. Nie Y, Liu Y, Luo Q and Huang S: Effect of dexmedetomidine combined with sufentanil for post-caesarean section intravenous analgesia: A randomised, placebo-controlled study. Eur J Anaesthesiol 31: 197-203, 2014.

31. Solca M: Acute pain management: Unmet needs and new advances in pain management. Eur J Anaesthesiol Suppl 25: 3-10, 2002.

32. Yagar S, Yavaş S and Karahalil B: The role of the ADRA2A C1291G genetic polymorphism in response to dexmedetomidine on patients undergoing coronary artery surgery. Mol Biol Rep 38: 3383-3391, 2011.

33. Rosmond R, Bouchard C and Björntorp P: A C-1291G polymorphism in the alpha2A-adrenergic receptor gene (ADRA2A) promoter is associated with cortisol escape from dexamethasone and elevated glucose levels. J Intern Med 251: 252-257, 2002.

34. Huang HC, Wu LS, Yu SC, Wu BJ, Lua AC, Lee SM and Liu CZ: The alpha-2A adrenergic receptor gene-1291C/G single nucleotide polymorphism is associated with the efficacy of methylphenidate in treating Taiwanese children and adolescents with attention-deficit hyperactivity disorder. Psychiatry Investig 15: 306-312, 2018.

(i) $($ ) This work is licensed under a Creative Commons Attribution-NonCommercial-NoDerivatives 4.0 International (CC BY-NC-ND 4.0) License. 